\title{
Plane wave discontinuous Galerkin methods for the 2D Helmholtz equation: analysis of the \$p\$-version
}

Article

Published Version

Hiptmair, R., Moiola, A. and Perugia, I. (2011) Plane wave discontinuous Galerkin methods for the 2D Helmholtz equation: analysis of the $\$ p \$$-version. SIAM Journal on Numerical Analysis (SINUM), 49 (1). pp. 264-284. ISSN 00361429 doi: https://doi.org/10.1137/090761057 Available at https://centaur.reading.ac.uk/28020/

It is advisable to refer to the publisher's version if you intend to cite from the work. See Guidance on citing.

To link to this article DOI: http://dx.doi.org/10.1137/090761057

Publisher: Society for Industrial and Applied Mathematics

All outputs in CentAUR are protected by Intellectual Property Rights law, including copyright law. Copyright and IPR is retained by the creators or other copyright holders. Terms and conditions for use of this material are defined in the End User Agreement.

www.reading.ac.uk/centaur 
Central Archive at the University of Reading

Reading's research outputs online 


\title{
PLANE WAVE DISCONTINUOUS GALERKIN METHODS FOR THE 2D HELMHOLTZ EQUATION: ANALYSIS OF THE $p$-VERSION*
}

\author{
R. HIPTMAIR ${ }^{\dagger}$, A. MOIOLA ${ }^{\dagger}$, AND I. PERUGIA $^{\ddagger}$
}

\begin{abstract}
Plane wave discontinuous Galerkin (PWDG) methods are a class of Trefftz-type methods for the spatial discretization of boundary value problems for the Helmholtz operator $-\Delta-$ $\omega^{2}, \omega>0$. They include the so-called ultra weak variational formulation from [O. Cessenat and B. Després, SIAM J. Numer. Anal., 35 (1998), pp. 255-299]. This paper is concerned with the a priori convergence analysis of PWDG in the case of $p$-refinement, that is, the study of the asymptotic behavior of relevant error norms as the number of plane wave directions in the local trial spaces is increased. For convex domains in two space dimensions, we derive convergence rates, employing mesh skeleton-based norms, duality techniques from [P. Monk and D. Wang, Comput. Methods Appl. Mech. Engrg., 175 (1999), pp. 121-136], and plane wave approximation theory.
\end{abstract}

Key words. Helmholtz equation, wave propagation, discontinuous Galerkin methods, plane waves, $p$-version error analysis, duality estimates

AMS subject classifications. 65N15, 65N30, 35J05

DOI. $10.1137 / 090761057$

1. Introduction. Standard low order Lagrangian finite element discretizations of boundary value problems for the Helmholtz equation $-\Delta u-\omega^{2} u=f$ are afflicted with the so-called pollution phenomenon [6]: though for sufficiently small $\omega h, h$ being the mesh size, an accurate approximation of $u$ is possible, the Galerkin procedure fails to provide it. Attempts to remedy this have focused on incorporating extra information in the form of plane wave functions $\boldsymbol{x} \mapsto \exp (i \omega \boldsymbol{d} \cdot \boldsymbol{x}),|\boldsymbol{d}|=1$, into the trial spaces. Prominent examples of such methods are the plane wave partition of unity finite element method of Babuška and Melenk [5] (see also [30]), the discontinuous enrichment method [4, 13, 32], the variational theory of complex rays (VTCR) [31], and the ultra weak variational formulation (UWVF) invented by Cessenat and Despres in the late 1990's [11]. Since then this latter method has seen rapid algorithmic development and extensions; see [21, 22, 23, 24, 25]. Even commercial simulation software has been based on it. ${ }^{1}$ This strongly motivates investigations into its theoretical foundations, to which the present paper aims to contribute.

It turns out that the UWVF can be recast as a special discontinuous Galerkin (DG) method employing local trial spaces spanned by a few plane waves, as pointed out in $[10,14,15]$. In a sense, this is a special case of a Trefftz-type approximation, as the local trial functions are solutions of the homogeneous Helmholtz equation $-\Delta u-$ $\omega^{2} u=0$. This perspective paves the way for marrying plane wave approximation with many of the various DG methods developed for second order elliptic boundary value problems. We have pursued this in $[15,20,28]$ for a class of primal and mixed DG methods, which generalize the ultra weak scheme, and which differ from each

* Received by the editors June 3, 2009; accepted for publication (in revised form) November 12, 2010; published electronically February 10, 2011.

http://www.siam.org/journals/sinum/49-1/76105.html

†SAM, ETH Zürich, CH-8092 Zürich, Switzerland (hiptmair@sam.math.ethz.ch, andrea.moiola@ sam.math.ethz.ch).

‡Dipartimento di Matematica, Università di Pavia, I-27100 Pavia, Italy (ilaria.perugia@unipv.it).

${ }^{1}$ See http://www.waveller.com/. 
other in the choice of the numerical fluxes; we refer to these methods as "plane wave discontinuous Galerkin (PWDG) methods."

In particular, in [15], an $h$-version error analysis for the PWDG method applied to the two dimensional (2D) inhomogeneous Helmholtz problem was carried out. In that case, independent of how many plane waves are used in the local approximation spaces, only first order convergence can be achieved in general. The analysis was restricted to a class of PWDG methods with flux parameters depending on the product wh (not including the classical UWVF of [11]). Key elements of this analysis are local approximation estimates and inverse estimates for plane waves, and a duality technique. This involves estimating the approximation error of the solution of an inhomogeneous dual problem by plane waves. High order convergence as $h \rightarrow 0$ is actually achieved in the homogeneous case $f \equiv 0$ only [28].

The application of a duality argument in the error analysis entails a threshold condition on the mesh size: quasi-optimality of the PWDG solution is guaranteed only if $\omega^{2} h$ is "sufficiently small"; see [15, Theorem 4.10]. In numerical experiments this is observed as a widening gap between discretization error and plane wave best approximation error. Thus, the notorious pollution effect that haunts local discretizations of wave propagation problems manifests itself in the theoretical estimates.

For polynomial schemes, their $p$-versions, also called the spectral approach, are immune to the pollution effect $[1,2,3]$. Thus, we believe that the spectral version $/ p$ version of PWDG, which strives for better accuracy by enlarging the local trial spaces, will also possess this desirable property. Besides, practical experience suggests that (well balanced local) p-refinement is highly advisable [25], because (local) smoothness/analyticity of the solution $u$ can be exploited. Ultimately, a judicious $h p$-refinement strategy will be the most attractive option, though one has to confront the notorious ill-conditioning of the linear systems arising from spectral PWDG approaches. Since aspects of implementation are not covered in this paper, we will gloss over this issue.

Unfortunately, a comprehensive $h p$-convergence analysis is elusive so far. Thus, the more modest aim of this paper is the derivation of $p$-version error estimates for the PWDG method applied to the 2D homogeneous Helmholtz equation. The used approach has little in common with the duality techniques pursued in $[15,28]$, because $p$-refinement does not yield any useful approximation of the solution of the inhomogeneous dual problem, since plane waves fail to approximate general functions.

Moreover, we cannot rely on coercivity in the seminorm of the bilinear form defining the PWDG method for general functions. Instead, we consider a weaker skeleton-based energy norm (i.e., containing interelement jump terms and boundary terms only) which is a norm on the space of local Trefftz functions only. We prove a coercivity result in this norm. This grants more freedom in the choice of the flux parameters; in particular, constant flux parameters are allowed so that also the classical UWVF of [11] is covered by our analysis.

Our argument is based on an estimate of the $L^{2}$-norm of Trefftz functions by their skeleton-based norm, which was discovered in the context of least squares Trefftz methods in [29]. We rederive this estimate in order to establish the dependence of the constants in front of the estimate explicitly not only on the mesh width $h$, but also on the wave number $\omega$. In parts, the analysis is carried out along the lines of [10]. On the other hand, we do not rewrite the PWDG bilinear form in terms of impedance traces, but stay closer to the DG setting, and our arguments are substantially simpler than those of [10]. 
We point out that the constant in front of the final $p$-version error estimates depends on the product $\omega h$. This is inevitable, because no accuracy can be expected unless the underlying wavelength is resolved by the trial space. Yet, in contrast to the $h$-version estimates of [10, sect. 4], the error bounds do not hinge on the assumption that $\omega h$ is "sufficiently small."

The abstract analysis contained in this paper could be extended directly to the three dimensional (3D) case. Also the elliptic regularity and the stability estimates for the inhomogeneous Helmholtz problem, obtained in [26] in the 2D case, have been proved also in three dimensions in [12] and [17]. $h$-version error estimates for the projection of homogeneous Helmholtz solutions onto plane wave spaces were obtained in [28] for the $2 \mathrm{D}$ case and then extended to the $3 \mathrm{D}$ case in [18] by using an argument which covers the $2 \mathrm{D}$ and $3 \mathrm{D}$ cases at once. The corresponding $p$-version estimates in the $3 \mathrm{D}$ case have been developed in the subsequent paper [19]. These results allow for a straightforward generalization of the $p$-version analysis of the PWDG method contained in this paper to the 3D Helmholtz equation.

The outline of this paper is as follows: In section 2, we report the derivation of the PWDG method for the homogeneous Helmholtz equation with impedance boundary conditions. Next, we derive $p$-version error estimates in section 3. They hinge on certain assumptions on the domain, mesh, and trial spaces, in particular the uniformity of element sizes. In section 3.1 we state a coercivity property and continuity of the PWDG bilinear form. Then, we prove quasi-optimality of the approximation error in a mesh skeleton-based norm and derive a bound for the $L^{2}$-norm of the error in section 3.2. There we follow [29] and [10]. Subsequently, from an approximation result proved in [18], we derive best approximation estimates in the skeleton-based norm in section 3.3. In section 3.4, we continue by stating energy-norm error estimates: these are reported in Theorem 3.15, which constitute the main result of the paper. In section 3.5, we derive error estimates in a stronger norm, also containing the difference between the gradient of the analytical solution and the gradient of a (computable) projection of the PWDG solution. The final section studies the PWDG discretization error numerically for some model problems.

2. The PWDG method. In this section, we introduce the plane wave discontinuous Galerkin (PWDG) method for the homogeneous Helmholtz equation, following [15].

Assume $\Omega$ to be a bounded Lipschitz domain in $\mathbb{R}^{d}, d=2,3$ (since the abstract error analysis developed in section 3 holds true in both two dimensions and three dimensions, we do not restrict to two dimensions at this point). For the duality argument used in our error analysis, we need to assume $\Omega$ to be convex.

Consider the Helmholtz boundary value problem

$$
\begin{array}{ll}
-\Delta u-\omega^{2} u=0 & \text { in } \Omega, \\
\nabla u \cdot \boldsymbol{n}+i \omega u=g & \text { on } \partial \Omega .
\end{array}
$$

Here, $\omega>0$ is a fixed wave number (the corresponding wavelength is $\lambda=2 \pi / \omega$ ), $\boldsymbol{n}$ is the outer normal unit vector to $\partial \Omega$, and $i$ is the imaginary unit. Inhomogeneous first order absorbing boundary conditions in the form of impedance boundary conditions are used in (2.1), with boundary data $g \in L^{2}(\partial \Omega)$.

Let $\mathcal{T}_{h}$ be a finite element partition of $\Omega$, with possible hanging nodes, of mesh width $h$ (i.e., $h=\max _{K \in \mathcal{T}_{h}} h_{K}$, with $\left.h_{K}:=\operatorname{diam}(K)\right)$ on which we define our PWDG method; we will denote by $\mathcal{F}_{h}=\bigcup_{K \in \mathcal{T}_{h}} \partial K$ the skeleton of the mesh, and set $\mathcal{F}_{h}^{B}=$ $\mathcal{F}_{h} \cap \partial \Omega$ and $\mathcal{F}_{h}^{I}=\mathcal{F}_{h} \backslash \mathcal{F}_{h}^{B}$. 
Consider an element $K$ of $\mathcal{T}_{h}$; we denote by $V_{p}(K)$ the plane wave space on $K$ spanned by $p$ plane waves, $p \in \mathbb{N}$ :

$$
V_{p}(K)=\left\{v \in L^{2}(K): v(\boldsymbol{x})=\sum_{j=1}^{p} \alpha_{j} \exp \left(i \omega \boldsymbol{d}_{j} \cdot \boldsymbol{x}\right), \alpha_{j} \in \mathbb{C}\right\},
$$

where $\boldsymbol{d}_{j},\left|\boldsymbol{d}_{j}\right|=1$, are $p$ different directions. Then, we define

$$
V_{p}\left(\mathcal{T}_{h}\right)=\left\{v \in L^{2}(\Omega):\left.v\right|_{K} \in V_{p}(K) \forall K \in \mathcal{T}_{h}\right\} .
$$

In the $p$-version setting, we assume the mesh $\mathcal{T}_{h}$ to be fixed, and we vary only p. Further assumptions on the problem domain, on the mesh $\mathcal{T}_{h}$, and on the approximation spaces $V_{p}\left(\mathcal{T}_{h}\right)$ will be made precise at the beginning of section 3 and in section 3.3 .

In order to derive the PWDG method, we start by writing problem (2.1) as a first order system:

$$
\begin{array}{ll}
i \omega \boldsymbol{\sigma}=\nabla u & \text { in } \Omega, \\
i \omega u-\nabla \cdot \boldsymbol{\sigma}=0 & \text { in } \Omega, \\
i \omega \boldsymbol{\sigma} \cdot \boldsymbol{n}+i \omega u=g & \text { on } \partial \Omega .
\end{array}
$$

By multiplying the first and second equation of (2.2) by smooth test functions $\boldsymbol{\tau}$ and $v$, respectively, and integrating by parts on each $K \in \mathcal{T}_{h}$, we obtain

$$
\begin{array}{ccc}
\int_{K} i \omega \boldsymbol{\sigma} \cdot \overline{\boldsymbol{\tau}} \mathrm{d} V+\int_{K} u \overline{\nabla \cdot \boldsymbol{\tau}} \mathrm{d} V-\int_{\partial K} u \overline{\boldsymbol{\tau} \cdot \boldsymbol{n}} \mathrm{d} S=0 & \forall \boldsymbol{\tau} \in \mathbf{H}(\mathrm{d} ; K), \\
\int_{K} i \omega u \bar{v} \mathrm{~d} V+\int_{K} \boldsymbol{\sigma} \cdot \overline{\nabla v} \mathrm{~d} V-\int_{\partial K} \boldsymbol{\sigma} \cdot \boldsymbol{n} \bar{v} \mathrm{~d} S=0 & \forall v \in H^{1}(K) .
\end{array}
$$

Replace $u, v$ by $u_{p}, v_{p} \in V_{p}\left(\mathcal{T}_{h}\right)$ and $\boldsymbol{\sigma}, \boldsymbol{\tau}$ by $\boldsymbol{\sigma}_{p}, \boldsymbol{\tau}_{p} \in V_{p}\left(\mathcal{T}_{h}\right)^{d}$. Then, approximate the traces of $u$ and $\sigma$ across interelement boundaries by the so-called numerical fluxes denoted by $\widehat{u}_{p}$ and $\widehat{\boldsymbol{\sigma}}_{p}$, respectively, to obtain

$$
\begin{aligned}
& \int_{K} i \omega \boldsymbol{\sigma}_{p} \cdot \overline{\boldsymbol{\tau}}_{p} \mathrm{~d} V+\int_{K} u_{p} \overline{\nabla \cdot \boldsymbol{\tau}_{p}} \mathrm{~d} V-\int_{\partial K} \widehat{u}_{p} \overline{\boldsymbol{\tau}_{p} \cdot \boldsymbol{n}} \mathrm{d} S=0 \quad \forall \boldsymbol{\tau}_{p} \in V_{p}(K)^{d}, \\
& \int_{K} i \omega u_{p} \bar{v}_{p} \mathrm{~d} V+\int_{K} \boldsymbol{\sigma}_{p} \cdot \overline{\nabla v}_{p} \mathrm{~d} V-\int_{\partial K} \widehat{\boldsymbol{\sigma}}_{p} \cdot \boldsymbol{n} \bar{v}_{p} \mathrm{~d} S=0 \quad \forall v_{p} \in V_{p}(K) .
\end{aligned}
$$

The numerical fluxes will be defined below; they also take into account the inhomogeneous boundary conditions.

Integrating again by parts the first equation of (2.4), we obtain

$$
\int_{K} \boldsymbol{\sigma}_{p} \cdot \overline{\boldsymbol{\tau}}_{p} \mathrm{~d} V=\frac{1}{i \omega} \int_{K} \nabla u_{p} \cdot \overline{\boldsymbol{\tau}}_{p} \mathrm{~d} V-\frac{1}{i \omega} \int_{\partial K}\left(u_{p}-\widehat{u}_{p}\right) \overline{\boldsymbol{\tau}_{p} \cdot \boldsymbol{n}} \mathrm{d} S .
$$

Since $\nabla_{h} V_{p}\left(\mathcal{T}_{h}\right) \subseteq V_{p}\left(\mathcal{T}_{h}\right)^{d}$, we can take $\boldsymbol{\tau}_{p}=\nabla v_{p}$ in each element. Inserting the resulting expression for $\int_{K} \boldsymbol{\sigma}_{p} \cdot \overline{\nabla v}_{p} \mathrm{~d} V$ into the second equation of (2.4), we arrive at

$$
\int_{K}\left(\nabla u_{p} \cdot \overline{\nabla v}_{p}-\omega^{2} u_{p} \bar{v}_{p}\right) \mathrm{d} V-\int_{\partial K}\left(u_{p}-\widehat{u}_{p}\right) \overline{\nabla v_{p} \cdot \boldsymbol{n}} \mathrm{d} S-\int_{\partial K} i \omega \widehat{\boldsymbol{\sigma}}_{p} \cdot \boldsymbol{n} \bar{v}_{p} \mathrm{~d} S=0 .
$$


Notice that the formulation (2.6) is equivalent to (2.4) in the sense that their $u_{p}$ solution components coincide and the $\boldsymbol{\sigma}_{p}$ solution component of (2.4) can be recovered from $u_{p}$ by using (2.5).

Another equivalent formulation can be obtained by integrating by parts once more the first term in (2.6) (notice that the boundary term appearing in this integration by parts cancels out with a boundary term already present in (2.6)):

$$
\int_{K}\left(\overline{-\Delta v_{p}-\omega^{2} v_{p}}\right) u_{p} \mathrm{~d} V+\int_{\partial K} \widehat{u}_{p} \overline{\nabla v_{p} \cdot \boldsymbol{n}} \mathrm{d} S-\int_{\partial K} i \omega \widehat{\boldsymbol{\sigma}}_{p} \cdot \boldsymbol{n} \bar{v}_{p} \mathrm{~d} S=0 .
$$

Since $v_{p} \in V_{p}\left(\mathcal{T}_{h}\right)$, the volume term in (2.7) vanishes; thus (2.7) simply becomes

$$
\int_{\partial K} \widehat{u}_{p} \overline{\nabla v_{p} \cdot \boldsymbol{n}} \mathrm{d} S-\int_{\partial K} i \omega \widehat{\boldsymbol{\sigma}}_{p} \cdot \boldsymbol{n} \bar{v}_{p} \mathrm{~d} S=0 .
$$

In order to define the numerical fluxes and complete the definition of the PWDG method, we recall some standard DG notation. Write $\boldsymbol{n}^{+}, \boldsymbol{n}^{-}$for the exterior unit normals on $\partial K^{+}$and $\partial K^{-}$, respectively. Let $u_{p}$ and $\boldsymbol{\sigma}_{p}$ be a piecewise smooth function and vector field on $\mathcal{T}_{h}$, respectively. On $\partial K^{-} \cap \partial K^{+}$, we define

$$
\begin{aligned}
\text { the averages: } & \left\{u_{p}\right\}:=\frac{1}{2}\left(u_{p}^{+}+u_{p}^{-}\right), \quad\left\{\left\{\boldsymbol{\sigma}_{p}\right\}:=\frac{1}{2}\left(\boldsymbol{\sigma}_{p}^{+}+\boldsymbol{\sigma}_{p}^{-}\right),\right. \\
\text {the jumps: } & \llbracket \boldsymbol{u}_{p} \rrbracket_{N}:=u_{p}^{+} \boldsymbol{n}^{+}+u_{p}^{-} \boldsymbol{n}^{-}, \quad \llbracket \boldsymbol{\sigma}_{p} \rrbracket_{N}:=\boldsymbol{\sigma}_{p}^{+} \cdot \boldsymbol{n}^{+}+\boldsymbol{\sigma}_{p}^{-} \cdot \boldsymbol{n}^{-} .
\end{aligned}
$$

Furthermore, we denote by $\nabla_{h}$ the elementwise application of $\nabla$. Then, we define the PWDG fluxes by setting

$$
\left\{\begin{array}{l}
\left.\widehat{\boldsymbol{\sigma}}_{p}=\frac{1}{i \omega} \llbracket\left\{\nabla_{h} u_{p}\right\}\right\}-\alpha \llbracket u_{p} \rrbracket_{N} \\
\widehat{u}_{p}=\left\{\left\{u_{p}\right\}-\beta \frac{1}{i \omega} \llbracket \nabla_{h} u_{p} \rrbracket_{N}\right.
\end{array}\right.
$$

on interior faces, and

$$
\left\{\begin{array}{l}
\widehat{\boldsymbol{\sigma}}_{p}=\frac{1}{i \omega} \nabla_{h} u_{p}-(1-\delta)\left(\frac{1}{i \omega} \nabla_{h} u_{p}+u_{p} \boldsymbol{n}-\frac{1}{i \omega} g \boldsymbol{n}\right) \\
\widehat{u}_{p}=u_{p}-\delta\left(\frac{1}{i \omega} \nabla_{h} u_{p} \cdot \boldsymbol{n}+u_{p}-\frac{1}{i \omega} g\right)
\end{array}\right.
$$

on boundary faces, where the parameters $\alpha, \beta$, and $\delta$ are the so-called flux parameters; assumptions on them will be specified in section 3 .

Adding (2.8) over all elements $K \in \mathcal{T}_{h}$ gives

$$
\begin{aligned}
\int_{\mathcal{F}_{h}^{I}}\left(\widehat{u}_{p} \llbracket \overline{\nabla_{h} v_{P}} \rrbracket_{N}-i \omega \widehat{\boldsymbol{\sigma}}_{p} \cdot \llbracket \bar{v}_{p} \rrbracket_{N}\right) \mathrm{d} S \\
\quad+\int_{\mathcal{F}_{h}^{B}}\left(\widehat{u}_{p} \overline{\nabla_{h} v_{P} \cdot \boldsymbol{n}}-i \omega \widehat{\boldsymbol{\sigma}}_{p} \cdot \boldsymbol{n} \bar{v}_{p}\right) \mathrm{d} S=0,
\end{aligned}
$$

and inserting the above defined numerical fluxes allows us to write the PWDG method as follows: find $u_{p} \in V_{p}\left(\mathcal{T}_{h}\right)$ such that, for all $v_{p} \in V_{p}\left(\mathcal{T}_{h}\right)$,

$$
\mathcal{A}_{h}\left(u_{p}, v_{p}\right)=\ell_{h}\left(v_{p}\right)
$$

Copyright (c) by SIAM. Unauthorized reproduction of this article is prohibited. 
where

$$
\begin{aligned}
\mathcal{A}_{h}(u, v)= & \left.\int_{\mathcal{F}_{h}^{I}}\{u\}\right\} \llbracket \overline{\nabla_{h} v} \rrbracket_{N} \mathrm{~d} S+i \omega^{-1} \int_{\mathcal{F}_{h}^{I}} \beta \llbracket \nabla_{h} u \rrbracket_{N} \llbracket \overline{\nabla_{h} v} \rrbracket_{N} \mathrm{~d} S \\
& -\int_{\mathcal{F}_{h}^{I}}\left\{\left\{\nabla_{h} u\right\} \cdot \llbracket \bar{v} \rrbracket_{N} \mathrm{~d} S+i \omega \int_{\mathcal{F}_{h}^{I}} \alpha \llbracket u \rrbracket_{N} \cdot \llbracket \bar{v} \rrbracket_{N} \mathrm{~d} S\right. \\
& +\int_{\mathcal{F}_{h}^{B}}(1-\delta) u \overline{\nabla_{h} v \cdot \boldsymbol{n}} \mathrm{d} S+i \omega^{-1} \int_{\mathcal{F}_{h}^{B}} \delta \nabla_{h} u \cdot \boldsymbol{n} \overline{\nabla_{h} v \cdot \boldsymbol{n}} \mathrm{d} S \\
& -\int_{\mathcal{F}_{h}^{B}} \delta \nabla_{h} u \cdot \boldsymbol{n} \bar{v} \mathrm{~d} S+i \omega \int_{\mathcal{F}_{h}^{B}}(1-\delta) u \bar{v} \mathrm{~d} S,
\end{aligned}
$$

and

$$
\ell_{h}(v)=i \omega^{-1} \int_{\mathcal{F}_{h}^{B}} \delta g \overline{\nabla_{h} v \cdot \boldsymbol{n}} \mathrm{d} S+\int_{\mathcal{F}_{h}^{B}}(1-\delta) g \bar{v} \mathrm{~d} S .
$$

The PWDG formulation is consistent by construction; thus, if $u \in H^{2}(\Omega)$ solves (2.1), then it holds that

$$
\mathcal{A}_{h}\left(u, v_{p}\right)=\ell_{h}\left(v_{p}\right) \quad \forall v_{p} \in V_{p}\left(\mathcal{T}_{h}\right) .
$$

3. Error analysis. We develop our a priori error analysis under the following additional assumptions:

Assumptions on the problem domain:

1. $\Omega$ is convex.

Assumptions on the mesh:

2. each element $K$ of $\mathcal{T}_{h}$ is a convex Lipschitz domain;

3. there exists a constant $\rho \in(0,1)$ such that each element $K \in \mathcal{T}_{h}$ contains a ball of radius $\rho h_{K}$ (shape regularity);

4. there exists a constant $\mu \in(0,1)$ such that, for each $K \in \mathcal{T}_{h}, h_{K} \geq \mu h$ (quasi-uniformity).

Assumptions on the flux parameters:

5. $\alpha, \beta$, and $\delta$ are real, strictly positive, and independent of $p, h$, and $\omega$, with $0<\delta \leq 1 / 2$.

REMARK 3.1. A choice of flux parameters that depends on $p$ and on the product $\omega$, in the spirit of standard DG methods and of the PWDG method of [15], will be discussed in Remark 3.16. The choice $\alpha=\beta=\delta=1 / 2$ gives rise to the original UWVF by Cessenat and Després (see [11] and [10]).

Define the broken Sobolev spaces

$$
H^{s}\left(\mathcal{T}_{h}\right)=\left\{w \in L^{2}(\Omega): w_{\left.\right|_{K}} \in H^{s}(K) \forall K \in \mathcal{T}_{h}\right\} .
$$

Let $T\left(\mathcal{T}_{h}\right)$ be the piecewise Trefftz space defined on $\mathcal{T}_{h}$ by

$$
T\left(\mathcal{T}_{h}\right)=\left\{w \in H^{2}\left(\mathcal{T}_{h}\right): \Delta w+\omega^{2} w=0 \text { in each } K \in \mathcal{T}_{h}\right\},
$$

and endow it with the norm (see Proposition 3.2)

$$
\begin{aligned}
\|\mid w\|_{\mathcal{F}_{h}}^{2}:= & \omega^{-1}\left\|\beta^{1 / 2} \llbracket \nabla_{h} w \rrbracket_{N}\right\|_{0, \mathcal{F}_{h}^{I}}^{2}+\omega\left\|\alpha^{1 / 2} \llbracket w \rrbracket_{N}\right\|_{0, \mathcal{F}_{h}^{I}}^{2} \\
& +\omega^{-1}\left\|\delta^{1 / 2} \nabla_{h} w \cdot \boldsymbol{n}\right\|_{0, \mathcal{F}_{h}^{B}}^{2}+\omega\left\|(1-\delta)^{1 / 2} w\right\|_{0, \mathcal{F}_{h}^{B}}^{2} .
\end{aligned}
$$

Copyright $\odot$ by SIAM. Unauthorized reproduction of this article is prohibited. 
In the following, we will also make use of the augmented norm

$$
\begin{aligned}
\left|\|w \mid\|_{\mathcal{F}_{h}^{+}}^{2}:=\right. & \left|\|w \mid\|_{\mathcal{F}_{h}}^{2}+\omega\left\|\beta^{-1 / 2}\{\{w\}\}\right\|_{0, \mathcal{F}_{h}^{I}}^{2}\right. \\
& +\omega^{-1}\left\|\alpha^{-1 / 2}\left\{\left\{\nabla_{h} w\right\}\right\}\right\|_{0, \mathcal{F}_{h}^{I}}^{2}+\omega\left\|\delta^{-1 / 2} w\right\|_{0, \mathcal{F}_{h}^{B}}^{2} .
\end{aligned}
$$

3.1. Auxiliary results. Here we collect technical prerequisites for the convergence analysis.

Proposition 3.2. The seminorm (3.1) is actually a norm on $T\left(\mathcal{T}_{h}\right)$.

Proof. Let $w \in T\left(\mathcal{T}_{h}\right)$ be such that $\||w|\|_{\mathcal{F}_{h}}^{2}=0$. Then $w \in H^{2}(\Omega)$ and satisfies $\Delta w+\omega^{2} w=0$ in $\Omega, w=0$, and $\nabla w \cdot \boldsymbol{n}=0$ on $\partial \Omega$, which implies $\nabla w \cdot \boldsymbol{n}+i \omega w=0$ on $\partial \Omega$. The uniqueness of the solution of problem (2.1) gives $w=0$.

Proposition 3.3. If $w \in T\left(\mathcal{T}_{h}\right)$, then

$$
\operatorname{Im}\left[\mathcal{A}_{h}(w, w)\right]=\left|\|w \mid\|_{\mathcal{F}_{h}}^{2} .\right.
$$

Proof. Provided that $u, v \in T\left(\mathcal{T}_{h}\right)$, local integration by parts permits us to rewrite the bilinear form $\mathcal{A}_{h}(u, v)$ as

$$
\begin{aligned}
\mathcal{A}_{h}(u, v)= & \left(\nabla_{h} u, \nabla_{h} v\right)_{0, \Omega}-\int_{\mathcal{F}_{h}^{\mathcal{T}}} \llbracket u \rrbracket_{N} \cdot\left\{\left\{\overline{\nabla_{h} v}\right\}\right\} \mathrm{d} S-\int_{\mathcal{F}_{h}^{\mathcal{I}}}\left\{\left\{\nabla_{h} u\right\} \cdot \llbracket \bar{v} \rrbracket_{N} \mathrm{~d} S\right. \\
& -\int_{\mathcal{F}_{h}^{\mathcal{B}}} \delta u \overline{\nabla_{h} v \cdot \boldsymbol{n}} \mathrm{d} S-\int_{\mathcal{F}_{h}^{\mathcal{B}}} \delta \nabla_{h} u \cdot \boldsymbol{n} \bar{v} \mathrm{~d} S \\
& +i \omega^{-1} \int_{\mathcal{F}_{h}^{\mathcal{I}}} \beta \llbracket \nabla_{h} u \rrbracket_{N} \llbracket \overline{\nabla_{h} v} \rrbracket_{N} \mathrm{~d} S+i \omega^{-1} \int_{\mathcal{F}_{h}^{\mathcal{B}}} \delta \nabla_{h} u \cdot \boldsymbol{n} \overline{\nabla_{h} v \cdot \boldsymbol{n}} \mathrm{d} S \\
& +i \omega \int_{\mathcal{F}_{h}^{\mathcal{I}}} \alpha \llbracket u \rrbracket_{N} \cdot \llbracket \bar{v} \rrbracket_{N} \mathrm{~d} S+i \omega \int_{\mathcal{F}_{h}^{\mathcal{B}}}(1-\delta) u \bar{v} \mathrm{~d} S-\omega^{2}(u, v)_{0, \Omega} .
\end{aligned}
$$

Therefore,

$$
\begin{aligned}
\mathcal{A}_{h}(w, w)= & \left\|\nabla_{h} w\right\|_{0, \Omega}^{2}-2 \operatorname{Re}\left[\int_{\mathcal{F}_{h}^{\mathcal{I}}} \llbracket w \rrbracket_{N} \cdot\left\{\left\{\overline{\nabla_{h} w}\right\}\right] \mathrm{d} S+\int_{\mathcal{F}_{h}^{\mathcal{B}}} \delta w \overline{\nabla_{h} w \cdot \boldsymbol{n}} \mathrm{d} S\right] \\
& +i \omega^{-1}\left\|\beta^{1 / 2} \llbracket \nabla_{h} w \rrbracket_{N}\right\|_{0, \mathcal{F}_{h}^{I}}^{2}+i \omega^{-1}\left\|\delta^{1 / 2} \nabla_{h} w \cdot \boldsymbol{n}\right\|_{0, \mathcal{F}_{h}^{B}}^{2} \\
& +i \omega\left\|\alpha^{1 / 2} \llbracket w \rrbracket_{N}\right\|_{0, \mathcal{F}_{h}^{I}}^{2}+i \omega\left\|(1-\delta)^{1 / 2} w\right\|_{0, \mathcal{F}_{h}^{B}}^{2}-\omega^{2}\|w\|_{0, \Omega}^{2},
\end{aligned}
$$

from which, by taking the imaginary part, we get the result.

REMARK 3.4. Well posedness of the PWDG method follows from Propositions 3.3 and 3.2. In fact, if $\mathcal{A}_{h}\left(u_{p}, v_{p}\right)=0$ for all $v_{p} \in V_{p}\left(\mathcal{T}_{h}\right)$, then $\mathcal{A}_{h}\left(u_{p}, u_{p}\right)=0$, and thus $\left\|\left|u_{p}\right|\right\|_{\mathcal{F}_{h}}=0$, which implies $u_{p}=0$.

Proposition 3.5. There exists a constant $C>0$ independent of $h, p$, and $\omega$ such that, for all $w_{1}, w_{2} \in H^{2}\left(\mathcal{T}_{h}\right)$,

$$
\left|\mathcal{A}_{h}\left(w_{1}, w_{2}\right)\right| \leq C||\left|w_{1}\right|||_{\mathcal{F}_{h}^{+}}||\left|w_{2}\right|||_{\mathcal{F}_{h}} .
$$

Proof. The result follows from the definition of $\mathcal{A}_{h}(\cdot, \cdot),(1-\delta)^{-1 / 2} \leq \delta^{-1 / 2}$, and repeated applications of the (weighted) Cauchy-Schwarz inequality.

3.2. Abstract error estimates. In the next proposition, we prove quasioptimality of the PWDG method in the $\||\cdot|\|_{\mathcal{F}_{h}}$-norm.

Copyright $@$ by SIAM. Unauthorized reproduction of this article is prohibited. 
Proposition 3.6. Let $u$ be the analytical solution to (2.1) and let $u_{p}$ be the $P W D G$ solution. Then, there exists a constant $C>0$ independent of $h, p$, and $\omega$ such that

$$
\left\||| u-\left.u_{p}||\right|_{\mathcal{F}_{h}} \leq\left. C \inf _{v_{p} \in V_{p}\left(\mathcal{T}_{h}\right)}\left|\| u-v_{p}\right|\right|_{\mathcal{F}_{h}^{+}}\right.
$$

where ||$|\cdot| \|_{\mathcal{F}_{h}^{+}}$is defined by (3.2).

Proof. We apply the triangle inequality and write

$$
||\left|u-u_{p}\right|||_{\mathcal{F}_{h}} \leq||\left|u-v_{p}\right|||_{\mathcal{F}_{h}}+||\left|u_{p}-v_{p}\right|||_{\mathcal{F}_{h}}
$$

for all $v_{p} \in V_{p}\left(\mathcal{T}_{h}\right)$. Since $u_{p}-v_{p} \in T\left(\mathcal{T}_{h}\right)$, Proposition 3.3 gives

$$
\left\|\left|u_{p}-v_{p}\right|\right\|_{\mathcal{F}_{h}}^{2}=\operatorname{Im}\left[\mathcal{A}_{h}\left(u_{p}-v_{p}, u_{p}-v_{p}\right)\right] .
$$

From Galerkin orthogonality and continuity of $\mathcal{A}_{h}(\cdot, \cdot)$ (see Proposition 3.5), we have

$$
\left.||\left|u_{p}-v_{p}\right|\right|_{\mathcal{F}_{h}} ^{2} \leq\left. C||\left|u-v_{p}\right|\right|_{\mathcal{F}_{h}^{+}}||\left|u_{p}-v_{p}\right|||_{\mathcal{F}_{h}},
$$

which, inserted into (3.3), gives the result.

Following [10, 29], we bound the $L^{2}$-norm of any Trefftz function by using a duality argument.

Lemma 3.7. There exists a constant $C>0$ independent of $h, p$, and $\omega$ such that, for any $w \in T\left(\mathcal{T}_{h}\right)$,

$$
\|w\|_{0, \Omega} \leq C \operatorname{diam}(\Omega)\left(\omega^{-1 / 2} h^{-1 / 2}+\omega^{1 / 2} h^{1 / 2}\right)\||w|\|_{\mathcal{F}_{h}} .
$$

Proof. Let $\varphi$ be in $L^{2}(\Omega)$. Consider the adjoint problem:

$$
\begin{array}{ll}
-\Delta v-\omega^{2} v=\varphi & \text { in } \Omega, \\
\nabla v \cdot \boldsymbol{n}-i \omega v=0 & \text { on } \partial \Omega
\end{array}
$$

The solution $v$ belongs to $H^{2}(\Omega)$, and since $\Omega$ is convex, the stability estimates

$$
\begin{aligned}
& |v|_{1, \Omega}+\omega\|v\|_{0, \Omega} \leq C_{1} \operatorname{diam}(\Omega)\|\varphi\|_{0, \Omega} \\
& |v|_{2, \Omega} \leq C_{2}(1+\omega \operatorname{diam}(\Omega))\|\varphi\|_{0, \Omega}
\end{aligned}
$$

hold, with $C_{1}, C_{2}>0$ depending only on the shape of $\Omega$ (see [26, Proposition 8.1.4], [12], and [17, Propositions 3.3, 3.5, and 3.6]).

Multiplying by $w \in T\left(\mathcal{T}_{h}\right)$, integrating by parts twice the first equation of (3.4) element by element (using $\Delta w+\omega^{2} w=0$ in each $K \in \mathcal{T}_{h}$ ), and taking into account

Copyright (C) by SIAM. Unauthorized reproduction of this article is prohibited. 
that $\nabla v \cdot \boldsymbol{n}=i \omega v$ on $\partial \Omega$, we obtain

$$
\begin{aligned}
\left|(w, \varphi)_{0, \Omega}\right|=\left|\sum_{K \in \mathcal{T}_{h}} \int_{\partial K}(\nabla w \cdot \boldsymbol{n} \bar{v}-w \overline{\nabla v \cdot \boldsymbol{n}}) \mathrm{d} S\right| \\
=\left|\int_{\mathcal{F}_{h}^{I}}\left(\llbracket \nabla_{h} w \rrbracket_{N} \bar{v}-\llbracket w \rrbracket_{N} \cdot \overline{\nabla v}\right) \mathrm{d} S+\int_{\mathcal{F}_{h}^{B}}\left(\nabla_{h} w \cdot \boldsymbol{n}+i \omega w\right) \bar{v} \mathrm{~d} S\right| \\
\leq \sum_{f \in \mathcal{F}_{h}^{I}}\left(\left\|\beta^{1 / 2} \llbracket \nabla_{h} w \rrbracket_{N}\right\|_{0, f}\left\|\beta^{-1 / 2} v\right\|_{0, f}+\left\|\alpha^{1 / 2} \llbracket w \rrbracket_{N}\right\|_{0, f}\left\|\alpha^{-1 / 2} \nabla_{h} v\right\|_{0, f}\right) \\
\quad+\sum_{f \in \mathcal{F}_{h}^{B}}\left(\left\|\delta^{1 / 2} \nabla w \cdot \boldsymbol{n}\right\|_{0, f}\left\|\delta^{-1 / 2} v\right\|_{0, f}+\omega^{1 / 2}\left\|\delta^{1 / 2} w\right\|_{0, f} \omega^{1 / 2}\left\|\delta^{-1 / 2} v\right\|_{0, f}\right) \\
\leq \mid\|w\|_{\mathcal{F}_{h}}\left[\sum_{f \in \mathcal{F}_{h}^{I}}\left(\omega\left\|\beta^{-1 / 2} v\right\|_{0, f}^{2}+\omega^{-1}\left\|\alpha^{-1 / 2} \nabla_{h} v\right\|_{0, f}^{2}\right)+\sum_{f \in \mathcal{F}_{h}^{B}} \omega\left\|\delta^{-1 / 2} v\right\|_{0, f}^{2}\right]^{1 / 2} \\
=:\left|\|w \mid\|_{\mathcal{F}_{h}} \mathcal{G}(v)^{1 / 2} .\right.
\end{aligned}
$$

Introducing, for convenience, a parameter $\gamma$ defined by $\gamma=\beta$ on interior faces and $\gamma=\delta$ on boundary faces, we have

$$
\mathcal{G}(v) \leq \sum_{K \in \mathcal{T}_{h}}\left(\omega\left\|\gamma^{-1 / 2} v\right\|_{0, \partial K}^{2}+\omega^{-1}\left\|\alpha^{-1 / 2} \nabla v\right\|_{0, \partial K}^{2}\right)
$$

We recall that, for any $K \in \mathcal{T}_{h}$, the trace inequality [9, Theorem 1.6.6]

$$
\|u\|_{0, \partial K}^{2} \leq C\|u\|_{0, K}\left(h_{K}^{-1}\|u\|_{0, K}+|u|_{1, K}\right) \quad \forall u \in H^{1}(K)
$$

holds with a constant $C>0$ depending only on the "shape regularity measure" $\rho$. Since $v \in H^{2}(\Omega)$, using the definition of the flux parameters, the trace estimate (3.6), the quasi-uniformity $\left(h_{K}^{-1} \leq \mu^{-1} h^{-1}\right)$, and the stability estimates (3.5), we can bound $\mathcal{G}(v)$ as follows:

$$
\begin{aligned}
\mathcal{G}(v) & \leq C \sum_{K \in \mathcal{T}_{h}}\left[\omega h_{K}^{-1}\|v\|_{0, K}^{2}+\omega\|v\|_{0, K}|v|_{1, K}+\omega^{-1} h_{K}^{-1}|v|_{1, K}^{2}+\omega^{-1}|v|_{1, K}|v|_{2, K}\right] \\
& \leq C \sum_{K \in \mathcal{T}_{h}} \mu^{-1}\left[\omega h^{-1}\|v\|_{0, K}^{2}+\omega h|v|_{1, K}^{2}+\omega^{-1} h^{-1}|v|_{1, K}^{2}+\omega^{-1} h|v|_{2, K}^{2}\right] \\
& \leq C\left[\operatorname{diam}(\Omega)^{2} \omega^{-1} h^{-1}+\operatorname{diam}(\Omega)^{2} \omega h+(1+\omega \operatorname{diam}(\Omega))^{2} \omega^{-1} h\right]\|\varphi\|_{0, \Omega}^{2} \\
& \leq C \operatorname{diam}(\Omega)^{2}\left(\omega^{-1} h^{-1}+\omega h\right)\|\varphi\|_{0, \Omega}^{2}
\end{aligned}
$$

(we have also used the obvious inequality $h \leq \operatorname{diam}(\Omega)$ ), with a constant $C>0$ independent of $h, p$, and $\omega$. Consequently, for all $\varphi \in L^{2}(\Omega)$, we obtain

$$
\frac{\left|(w, \varphi)_{0, \Omega}\right|}{\|\varphi\|_{0, \Omega}} \leq C \operatorname{diam}(\Omega)\left(\omega^{-1 / 2} h^{-1 / 2}+\omega^{1 / 2} h^{1 / 2}\right)\left|\|w \mid\|_{\mathcal{F}_{h}}\right.
$$

and the result readily follows.

By applying Lemma 3.7 to $u-u_{p} \in T\left(\mathcal{T}_{h}\right)$ we can bound the $L^{2}$-norm of the error by its $\left.|| \cdot||\right|_{\mathcal{F}_{h}}$-norm, like in [10].

Copyright (C) by SIAM. Unauthorized reproduction of this article is prohibited. 
Corollary 3.8. Let $u$ be the analytical solution to (2.1) and let $u_{p}$ be the PWDG solution. Then, there exists a constant $C>0$ independent of $h, p$, and $\omega$ such that

$$
\left\|u-u_{p}\right\|_{0, \Omega} \leq C \operatorname{diam}(\Omega)\left(\omega^{-1 / 2} h^{-1 / 2}+\omega^{1 / 2} h^{1 / 2}\right)\left\|\left|u-u_{p}\right|\right\|_{\mathcal{F}_{h}} .
$$

3.3. Best approximation estimates. From now on, we restrict ourselves to the $2 \mathrm{D}$ case $d=2$. We also make the following assumptions on the approximation spaces:

(i) $p=2 m+1$, with $m \in \mathbb{N} \backslash\{0\}$, is constant;

(ii) the $p$ directions $\left\{\boldsymbol{d}_{\ell}=\left(\cos \theta_{\ell}, \sin \theta_{\ell}\right)\right\}_{\ell=-m}^{m}$ satisfy the following condition: there exists $\eta \in(0,1]$ such that the minimum angle between two different directions is greater than or equal to $2 \pi \eta / p$. by

Given a domain $D \subset \mathbb{R}^{2}$, let $\|\cdot\|_{s, \omega, D}$ be the $\omega$-weighted Sobolev norm defined

$$
\|v\|_{s, \omega, D}^{2}=\sum_{j=0}^{s} \omega^{2(s-j)}|v|_{j, D}^{2} .
$$

We quote the following technical result proved in [18] by means of Vekua theory and approximation estimates for harmonic polynomials. For large $p$, the orders of convergence are summarized in much simpler form in Remark 3.13.

TheOREm 3.9. Let $D \subset \mathbb{R}^{2}$ be a bounded, convex Lipschitz domain of diameter $h_{D}$ which contains a ball of radius $\rho h_{D}$, with $0<\rho \leq 1 / 2$. Let $u \in H^{k+1}(D), k \geq 0$, be such that $\Delta u+\omega^{2} u=0$ in $D$. Fix $m \geq 1$ and assume the directions $\left\{\boldsymbol{d}_{\ell}\right\}_{\ell=-m}^{m}$ to satisfy the minimum angle assumption stated above.

Finally, assume that

$$
k \leq\left\lceil\frac{m+1}{2}\right\rceil
$$

Then, there exist $\sigma_{1}, \ldots, \sigma_{p} \in \mathbb{C}$ such that if we set $P_{\omega} u:=\sum_{\ell=1}^{p} \sigma_{\ell} e^{i \omega \boldsymbol{x} \cdot \boldsymbol{d}_{\ell}}$, for every $0 \leq j \leq k$ we have

$$
\begin{aligned}
\left\|u-P_{\omega} u\right\|_{j, \omega, D} \leq C e^{(7 / 4-3 / 4 \rho) \omega h_{D}}\left(1+\left(\omega h_{D}\right)^{j+8}\right) h_{D}^{k+1-j} \\
\qquad \cdot\left[\left(\frac{\log \left(k+1+\left\lfloor\frac{m+1}{2}\right\rfloor\right)}{k+1+\left\lfloor\frac{m+1}{2}\right\rfloor}\right)^{k+1-j}+\left(\frac{e^{5 / 2}}{2 \eta^{2} \rho^{1 / 2}} \frac{1+\omega h_{D}}{\sqrt{m+1}}\right)^{m}\right]\|u\|_{k+1, \omega, D},
\end{aligned}
$$

with the constant $C>0$ depending only on the shape of $D, j$, and $k$, but not on $h_{D}$, $p, \omega, \eta$, and $u$.

We point out that, thanks to the assumptions made earlier, Theorem 3.9 can be applied to the elements $K \in \mathcal{T}_{h}$. Also note that $p=2 m+1$ is the spectral discretization parameter.

Set, for simplicity,

$$
\mathcal{L}(k, m)=\frac{\log \left(k+1+\left\lfloor\frac{m+1}{2}\right\rfloor\right)}{k+1+\left\lfloor\frac{m+1}{2}\right\rfloor} ;
$$

notice that $0<\mathcal{L}(k, m)<1$. 
LEMMA 3.10. We have the following estimates:

$$
\begin{aligned}
\omega \| u- & P_{\omega} u \|_{0, \mathcal{F}_{h}}^{2} \\
\leq & C e^{(7 / 2-3 / 2 \rho) \omega h}\left(1+(\omega h)^{17}\right) \omega h^{2 k+1}\left[\mathcal{L}(k, m)^{k+1}+\left(\frac{e^{5 / 2}}{2 \eta^{2} \rho^{1 / 2}} \frac{1+\omega h}{\sqrt{m+1}}\right)^{m}\right] \\
\cdot & {\left[\mathcal{L}(k, m)^{k}+\left(\frac{e^{5 / 2}}{2 \eta^{2} \rho^{1 / 2}} \frac{1+\omega h}{\sqrt{m+1}}\right)^{m}\right]\|u\|_{k+1, \omega, \Omega}^{2}, }
\end{aligned}
$$

$$
\begin{aligned}
\omega^{-1}\left\|\nabla_{h}\left(u-P_{\omega} u\right)\right\|_{0, \mathcal{F}_{h}}^{2} & \\
\leq C & e^{(7 / 2-3 / 2 \rho) \omega h}\left(1+(\omega h)^{19}\right) \omega^{-1} h^{2 k-1}\left[\mathcal{L}(k, m)^{k}+\left(\frac{e^{5 / 2}}{2 \eta^{2} \rho^{1 / 2}} \frac{1+\omega h}{\sqrt{m+1}}\right)^{m}\right] \\
\cdot & {\left[\mathcal{L}(k, m)^{k-1}+\left(\frac{e^{5 / 2}}{2 \eta^{2} \rho^{1 / 2}} \frac{1+\omega h}{\sqrt{m+1}}\right)^{m}\right]\|u\|_{k+1, \omega, \Omega}^{2}, }
\end{aligned}
$$

with the constant $C>0$ independent of $h, p, \omega$, and $u$.

Proof. Using (3.6), Theorem 3.9, the fact that $(1+\omega h)>1$, and $\mathcal{L}(k, m)<1$, we have

$$
\begin{aligned}
&\left\|u-P_{\omega} u\right\|_{0, \partial K}^{2} \leq C\left(h_{K}^{-1}\left\|u-P_{\omega} u\right\|_{0, K}^{2}+\left\|u-P_{\omega} u\right\|_{0, K}\left|u-P_{\omega} u\right|_{1, K}\right) \\
& \leq C e^{(7 / 2-3 / 2 \rho) \omega h_{K}}\left(1+\left(\omega h_{K}\right)^{17}\right) h_{K}^{2 k+1}\left[\mathcal{L}(k, m)^{k+1}+\left(\frac{e^{5 / 2}}{2 \eta^{2} \rho^{1 / 2}} \frac{1+\omega h_{K}}{\sqrt{m+1}}\right)^{m}\right] \\
& \cdot\left[\mathcal{L}(k, m)^{k}+\left(\frac{e^{5 / 2}}{2 \eta^{2} \rho^{1 / 2}} \frac{1+\omega h_{K}}{\sqrt{m+1}}\right)^{m}\right]\|u\|_{k+1, \omega, K}^{2}
\end{aligned}
$$

and

$$
\begin{aligned}
\left\|\nabla\left(u-P_{\omega} u\right)\right\|_{0, \partial K}^{2} \leq C\left(h_{K}^{-1}\left|u-P_{\omega} u\right|_{1, K}^{2}+\left|u-P_{\omega} u\right|_{1, K}\left|u-P_{\omega} u\right|_{2, K}\right) \\
\leq C e^{(7 / 2-3 / 2 \rho) \omega h_{K}}\left(1+\left(\omega h_{K}\right)^{19}\right) h_{K}^{2 k-1}\left[\mathcal{L}(k, m)^{k}+\left(\frac{e^{5 / 2}}{2 \eta^{2} \rho^{1 / 2}} \frac{1+\omega h_{K}}{\sqrt{m+1}}\right)^{m}\right] \\
\cdot\left[\mathcal{L}(k, m)^{k-1}+\left(\frac{e^{5 / 2}}{2 \eta^{2} \rho^{1 / 2}} \frac{1+\omega h_{K}}{\sqrt{m+1}}\right)^{m}\right]\|u\|_{k+1, \omega, K}^{2} \cdot
\end{aligned}
$$

Adding over all elements and multiplying by $\omega^{ \pm 1}$ gives the two bounds.

The following theorem is a straightforward consequence of Lemma 3.10.

THEOREM 3.11. We have the following estimate:

$$
\begin{aligned}
& \|\| u-P_{\omega} u\|\|_{\mathcal{F}_{h}^{+}}^{2} \leq C h^{2 k} \\
& \left\{\omega^{-1} h^{-1}\left[\mathcal{L}(k, m)^{k+1}+\left(\frac{e^{5 / 2}}{2 \eta^{2} \rho^{1 / 2}} \frac{1+\omega h}{\sqrt{m+1}}\right)^{m}\right]\left[\mathcal{L}(k, m)^{k}+\left(\frac{e^{5 / 2}}{2 \eta^{2} \rho^{1 / 2}} \frac{1+\omega h}{\sqrt{m+1}}\right)^{m}\right]\right. \\
& \left.+\left[\mathcal{L}(k, m)^{k}+\left(\frac{e^{5 / 2}}{2 \eta^{2} \rho^{1 / 2}} \frac{1+\omega h}{\sqrt{m+1}}\right)^{m}\right]\left[\mathcal{L}(k, m)^{k-1}+\left(\frac{e^{5 / 2}}{2 \eta^{2} \rho^{1 / 2}} \frac{1+\omega h}{\sqrt{m+1}}\right)^{m}\right]\right\} \\
& \cdot\|u\|_{k+1, \omega, \Omega}^{2},
\end{aligned}
$$

Copyright $@$ by SIAM. Unauthorized reproduction of this article is prohibited. 

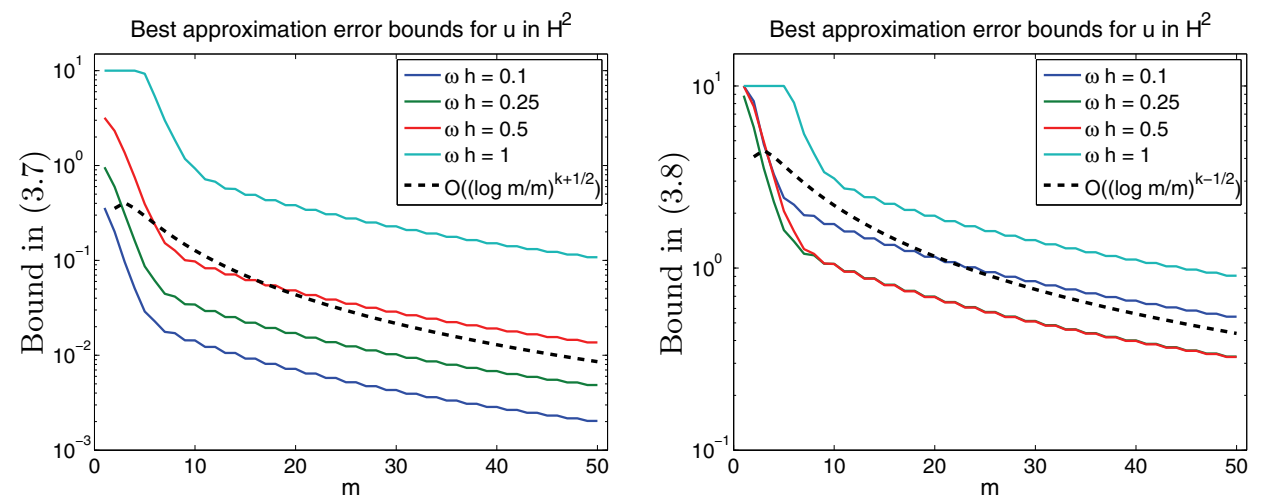

FIG. 3.1. Qualitative plots of the bounds given in Lemma 3.10 (with cutoff at 10).

with $C=C(\omega h)>0$ independent of $p$ and $u$, but increasing as a function of the product $\omega$ h.

REMARK 3.12. The graphs of the bounds of Lemma 3.10 given in Figure 3.1 highlight the pronounced increase of the constants for large $\omega$ h and small $p$. This is evidence of a threshold condition, that is, a minimal resolution requirement on the plane wave space before any reasonable approximation can be expected.

REMARK 3.13. For $k \leq\left\lceil\frac{m+1}{2}\right\rceil$ (see Theorem 3.9), $\mathcal{L}(k, m)$ asymptotically behaves, for increasing $m$, like $\log (m) / m$ (and thus like $\log (p) / p$ ) (see Figure 3.1), whereas the term $\left(\frac{C}{\sqrt{m+1}}\right)^{m}$ decays faster than exponentially. Therefore, the estimate of Theorem 3.11, for large $p$, can be written as

$$
\left\|\mid u-P_{\omega} u\right\|_{\mathcal{F}_{h}^{+}} \leq C \omega^{-1 / 2} h^{k-1 / 2}\left(\frac{\log (p)}{p}\right)^{k-1 / 2}\|u\|_{k+1, \omega, \Omega}
$$

Due to Lemma 3.10, the orders of convergence are not improved when working with the weaker $\||\cdot|\|_{\mathcal{F}_{h}}$-norm.

REMARK 3.14. If the function $u$ to be approximated is regular enough such that it can be extended analytically to a strictly larger domain, the convergence with respect to $p$ turns out to be exponential. Indeed, the algebraic term in the assertion of Theorem 3.9 can be replaced with an exponential one provided by Corollary 2.7 of [27] or Theorem 6.3 .3 of [8]. There the results refer to the approximation of holomorphic functions by complex polynomials, but they carry over to the approximation by plane waves. The speed of exponential convergence depends on how far u can be extended analytically.

This fact also implies that in elements of $\mathcal{T}_{h}$ that have a positive distance from $\partial \Omega$, we obtain exponential convergence in $p$ for the local best approximation by plane waves.

3.4. Error estimates. In the following theorem, we state error estimates for the PWDG method in the following energy-type norm:

$$
\|w\|_{D G}^{2}:=\left|\|w \mid\|_{\mathcal{F}_{h}}^{2}+\omega^{2}\|w\|_{0, \Omega}^{2} .\right.
$$

THEOREM 3.15. Let $u \in H^{k+1}(\Omega)$ be the analytical solution to (2.1) and let $u_{p}$ be the PWDG solution. For $p$ sufficiently large, there exists a $C=C(\omega h)>0$ 
independent of $p$ and $u$, but increasing as a function of the product wh, such that

$$
\begin{gathered}
\|\| u-u_{p}\|\|_{\mathcal{F}_{h}} \leq C \omega^{-1 / 2} h^{k-1 / 2}\left(\frac{\log (p)}{p}\right)^{k-1 / 2}\|u\|_{k+1, \omega, \Omega}, \\
\omega\left\|u-u_{p}\right\|_{0, \Omega} \leq C \operatorname{diam}(\Omega) h^{k-1}\left(\frac{\log (p)}{p}\right)^{k-1 / 2}\|u\|_{k+1, \omega, \Omega},
\end{gathered}
$$

and thus

$$
\begin{aligned}
& \left\|u-u_{p}\right\|_{D G} \\
& \quad \leq C \operatorname{diam}(\Omega)^{1 / 2}\left[\omega^{-1 / 2}+\operatorname{diam}(\Omega)^{1 / 2}\right] h^{k-1}\left(\frac{\log (p)}{p}\right)^{k-1 / 2}\|u\|_{k+1, \omega, \Omega} .
\end{aligned}
$$

Proof. The first two bounds follow from Proposition 3.6, Remark 3.13, and Corollary 3.8. The third bound is a direct consequence of the first two.

REMARK 3.16. If we choose the flux parameters depending on $p$ and wh in the following way,

$$
\alpha=\frac{\mathrm{a}}{\omega h} \frac{p}{\log (p)}, \quad \beta=\mathrm{b} \omega h \frac{\log (p)}{p}, \quad \delta=\mathrm{d} \omega h \frac{\log (p)}{p},
$$

with $\mathrm{a}, \mathrm{b}$, and $\mathrm{d}$ strictly positive and independent of $h, \omega$, and $p$, again with $0<\delta \leq$ $1 / 2$, the result of Lemma 3.7 becomes

$$
\|w\|_{0, \Omega} \leq C \operatorname{diam}(\Omega)\left[\left(\frac{p}{\log (p)}\right)^{1 / 2} \omega^{-1} h^{-1}+\left(\frac{p}{\log (p)}\right)^{1 / 2}+\omega h\right]\|\| w \|_{\mathcal{F}_{h}},
$$

and the best approximation estimate of Theorem 3.11 is

$$
\begin{aligned}
& \|\| u-P_{\omega} u \|_{\mathcal{F}_{h}^{+}}^{2} \leq C h^{2 k} \\
& \left\{\frac{p}{\log (p)}\left[\mathcal{L}(k, m)^{k+1}+\left(\frac{e^{5 / 2}}{2 \eta^{2} \rho^{1 / 2}} \frac{1+\omega h}{\sqrt{m+1}}\right)^{m}\right]\left[\mathcal{L}(k, m)^{k}+\left(\frac{e^{5 / 2}}{2 \eta^{2} \rho^{1 / 2}} \frac{1+\omega h}{\sqrt{m+1}}\right)^{m}\right]\right. \\
& \left.+\frac{\log (p)}{p}\left[\mathcal{L}(k, m)^{k}+\left(\frac{e^{5 / 2}}{2 \eta^{2} \rho^{1 / 2}} \frac{1+\omega h}{\sqrt{m+1}}\right)^{m}\right]\left[\mathcal{L}(k, m)^{k-1}+\left(\frac{e^{5 / 2}}{2 \eta^{2} \rho^{1 / 2}} \frac{1+\omega h}{\sqrt{m+1}}\right)^{m}\right]\right\} \\
& \cdot\|u\|_{k+1, \omega, \Omega}^{2} ;
\end{aligned}
$$

consequently, reasoning as in Remark 3.13 yields the estimate

$$
\|\| u-P_{\omega} u\|\|_{\mathcal{F}_{h}^{+}} \leq C h^{k}\left(\frac{\log (p)}{p}\right)^{k}\|u\|_{k+1, \omega, \Omega}
$$

where full order $k$ for the best approximation is achieved. On the other hand, the final error bounds (see Theorem 3.15) for this choice of flux parameters are

$$
\begin{aligned}
&\|\| u-u_{p}\|\|_{\mathcal{F}_{h}} \leq C h^{k}\left(\frac{\log (p)}{p}\right)^{k}\|u\|_{k+1, \omega, \Omega}, \\
& \omega\left\|u-u_{p}\right\|_{0, \Omega} \leq C \operatorname{diam}(\Omega) h^{k-1}\left(\frac{\log (p)}{p}\right)^{k-1 / 2}\|u\|_{k+1, \omega, \Omega}, \\
&\left\|u-u_{p}\right\|_{D G} \leq C \operatorname{diam}(\Omega) h^{k-1}\left(\frac{\log (p)}{p}\right)^{k-1 / 2}\|u\|_{k+1, \omega, \Omega} .
\end{aligned}
$$

Copyright (c) by SIAM. Unauthorized reproduction of this article is prohibited. 
Thus, the gain of half a power of $\log (p) / p$ in the best approximation estimate, with respect to the case of constant flux parameters, is compensated by a loss of half a power of $\log (p) / p$ in the result of Lemma 3.7, and thus the order of convergence in the energy-norm is the same as in the case of constant flux parameters.

REMARK 3.17. Matching the final estimate of Theorem 3.15 with the best approximation estimate (3.9), we find that the bounds feature optimal asymptotic behavior with respect to $p$, but half a power of $h$ is lost.

Remark 3.18. The proof of the "coercivity" result of Proposition 3.3 does not involve inverse trace inequalities. This allows us to choose either constant flux parameters or the variable flux parameters discussed in Remark 3.16, which, in both cases, give convergence in the energy-norm of order $(\log (p) / p)^{k-1 / 2}$.

On the other hand, the bound of the $L^{2}$-norm of the trace of a discrete function on the boundary of an element $K$ by the $L^{2}$-norm of the discrete function within $K$ involves a constant proportional to $p h_{K}^{-1 / 2}$ (see numerical evidence in [15]). Therefore, the use of inverse trace inequalities would have required a choice of the flux parameters similar to the one in Remark 3.16 , but with $p^{2}$ instead of $p / \log (p)$, resulting in a deterioration of the order of convergence of the energy-norm by a factor $p \log (p)$.

3.5. Error estimates in stronger norms. It would be desirable to derive an asymptotically quasi-optimal estimate of $\left\|\nabla_{h}\left(u-u_{p}\right)\right\|_{0, \Omega}$ as was achieved for the $h$ version of PWDG in [15]. The duality technique employed in our approach does not provide such estimates. We have to settle for weaker results.

Define the following $H^{1}\left(\mathcal{T}_{h}\right)$-orthogonal projection onto the space $\mathbb{P}^{p}\left(\mathcal{T}_{h}\right) \subset$ $H^{1}(\Omega)$ of globally continuous, $\mathcal{T}_{h}$-piecewise polynomial functions of degree $\leq p$ : $\mathcal{P}: H^{1}\left(\mathcal{T}_{h}\right) \rightarrow \mathbb{P}^{p}\left(\mathcal{T}_{h}\right)$ is such that, if $w \in H^{1}\left(\mathcal{T}_{h}\right)$,

$$
\mathcal{L}_{h}(\mathcal{P}(w), v)=\mathcal{L}_{h}(w, v) \quad \forall v \in \mathbb{P}^{p}\left(\mathcal{T}_{h}\right)
$$

where

$$
\mathcal{L}_{h}(w, v):=\int_{\Omega}\left(\nabla_{h} w \cdot \overline{\nabla_{h} v}+\omega^{2} w \bar{v}\right) d V \quad \forall w, v \in H^{1}\left(\mathcal{T}_{h}\right)
$$

Note that, given $w$, the computation of $\mathcal{P}$ amounts to solving a Neumann boundary value problem for $-\Delta+\omega^{2}$ by means of $p$-degree Lagrangian finite elements. Thus, in principle, $\mathcal{P} u_{p}$ can be obtained from the PWDG solution $u_{p} \in V_{p}\left(\mathcal{T}_{h}\right)$ by means of solving a discrete positive definite second order elliptic boundary value problem in a postprocessing step.

Proposition 3.19. With the same assumptions as in Theorem 3.15, we have

$$
\left\|\nabla\left(u-\mathcal{P}\left(u_{p}\right)\right)\right\|_{0, \Omega} \leq C\left(\operatorname{diam}(\Omega)+\omega^{-1}\right) h^{k-1}\left(\frac{\log (p)}{p}\right)^{k-1 / 2}\|u\|_{k+1, \omega, \Omega}
$$

with $C=C(\omega h)>0$ independent of $p$ and $u$, but depending monotonically on the product $\omega$ h.

Proof. By the triangle inequality, we can write

$$
\left\|\nabla\left(u-\mathcal{P}\left(u_{p}\right)\right)\right\|_{0, \Omega} \leq\|\nabla(u-\mathcal{P}(u))\|_{0, \Omega}+\left\|\nabla\left(\mathcal{P}\left(u-u_{p}\right)\right)\right\|_{0, \Omega} .
$$

Copyright $@$ ㅇ by SIAM. Unauthorized reproduction of this article is prohibited. 
We bound the second term on the right-hand side. By the definition of $\mathcal{P}$, for all $v \in \mathbb{P}^{p}\left(\mathcal{T}_{h}\right)$, local integration by parts gives

$$
\begin{aligned}
& \mathcal{L}_{h}\left(\mathcal{P}\left(u-u_{p}\right), v\right)=\sum_{K \in \mathcal{T}_{h}} \int_{K} \nabla\left(u-u_{p}\right) \cdot \overline{\nabla v} \mathrm{~d} V+\omega^{2}\left(u-u_{p}, v\right)_{0, \Omega} \\
& =-\sum_{K \in \mathcal{T}_{h}} \int_{K} \Delta\left(u-u_{p}\right) \bar{v} \mathrm{~d} V+\sum_{K \in \mathcal{T}_{h}} \int_{\partial K} \nabla\left(u-u_{p}\right) \cdot \boldsymbol{n}_{K} \bar{v} \mathrm{~d} S+\omega^{2}\left(u-u_{p}, v\right)_{0, \Omega} \\
& =2 \omega^{2}\left(u-u_{p}, v\right)_{0, \Omega}+\int_{\mathcal{F}_{h}^{I}} \llbracket \nabla_{h}\left(u-u_{p}\right) \rrbracket_{N} \bar{v} \mathrm{~d} S+\int_{\mathcal{F}_{h}^{B}} \nabla_{h}\left(u-u_{p}\right) \cdot \boldsymbol{n} \bar{v} \mathrm{~d} S .
\end{aligned}
$$

Aiming for the ||$|w|||_{\mathcal{F}_{h}}$-norm, we use the Cauchy-Schwarz inequality and get

$$
\begin{aligned}
\mathcal{L}_{h}\left(\mathcal{P}\left(u-u_{p}\right), v\right) & \leq 2 \omega\left\|u-u_{p}\right\|_{0, \Omega} \omega\|v\|_{0, \Omega} \\
& +\omega^{-1 / 2}\left\|\beta^{1 / 2} \llbracket \nabla_{h}\left(u-u_{p}\right) \rrbracket_{N}\right\|_{0, \mathcal{F}_{h}^{I}} \omega^{1 / 2}\left\|\beta^{-1 / 2} v\right\|_{0, \mathcal{F}_{h}^{I}} \\
& +\omega^{-1 / 2}\left\|\delta^{1 / 2} \nabla_{h}\left(u-u_{p}\right) \cdot \boldsymbol{n}\right\|_{0, \mathcal{F}_{h}^{B}} \omega^{1 / 2}\left\|\delta^{-1 / 2} v\right\|_{0, \mathcal{F}_{h}^{B}} \\
& \leq 2 \omega\left\|u-u_{p}\right\|_{0, \Omega} \omega\|v\|_{0, \Omega} \\
& +\|\| u-u_{p}\|\|_{\mathcal{F}_{h}} \omega^{1 / 2} \max \left\{\delta^{-1 / 2}, \beta^{-1 / 2}\right\}\|v\|_{0, \mathcal{F}_{h}} .
\end{aligned}
$$

Now, the trace inequality (3.6) gives

$$
\begin{aligned}
\mathcal{L}_{h}\left(\mathcal{P}\left(u-u_{p}\right), v\right) \leq & 2 \omega\left\|u-u_{p}\right\|_{0, \Omega} \omega\|v\|_{0, \Omega}+C(\omega h)^{-1 / 2}\|\| u-u_{p}\|\|_{\mathcal{F}_{h}} \\
& \cdot \max \left\{\delta^{-1 / 2}, \beta^{-1 / 2}\right\} \cdot\left(\omega\|v\|_{0, \Omega}+\omega h\|\nabla v\|_{0, \Omega}\right) \\
\leq & \left(\omega^{2}\left\|u-u_{p}\right\|_{0, \Omega}^{2}+(\omega h)^{-1}\left\|u-u_{p}\right\| \|_{\mathcal{F}_{h}}^{2}\right)^{1 / 2} \\
& \cdot C \max \left\{\delta^{-1 / 2}, \beta^{-1 / 2}\right\} \max \{\omega h, 1\} \\
& \cdot\left(\omega^{2}\|v\|_{0 . \Omega}^{2}+\|\nabla v\|_{0, \Omega}^{2}\right)^{1 / 2}
\end{aligned}
$$

where $C>0$ depends only on the shape-regularity of the mesh $\mathcal{T}_{h}$. Setting $v:=$ $\mathcal{P}\left(u-u_{p}\right)$ yields the estimate

$$
\begin{aligned}
\left\|\nabla \mathcal{P}\left(u-u_{p}\right)\right\|_{0, \Omega}^{2}+\omega^{2}\left\|\mathcal{P}\left(u-u_{p}\right)\right\|_{0 . \Omega}^{2} \leq & C(\min \{\delta, \beta\})^{-1} \max \{\omega h, 1\}^{2} \\
\cdot & \cdot\left(\omega^{2}\left\|u-u_{p}\right\|_{0, \Omega}^{2}+(\omega h)^{-1}\|\|-u_{p} \|_{\mathcal{F}_{h}}^{2}\right) .
\end{aligned}
$$

We plug in the estimates of Theorem 3.15 and allow $C>0$ to depend on an upper bound for $\omega h$ and also on the (constant) flux parameters. Thus, we arrive at

$$
\left\|\nabla \mathcal{P}\left(u-u_{p}\right)\right\|_{0, \Omega} \leq C\left(\operatorname{diam}(\Omega)+\omega^{-1}\right) h^{k-1}\left(\frac{\log (p)}{p}\right)^{k-1 / 2}\|u\|_{k+1, \omega, \Omega} .
$$

Further, standard error estimates for $H^{1}$-conforming Lagrangian finite element spaces [7] provide

$$
\|\nabla(u-\mathcal{P} u)\|_{0, \Omega} \leq C \frac{h^{k}}{p^{k}}\|u\|_{k+1, \omega, \Omega},
$$

where $C>0$ depends on the shape-regularity of $\mathcal{T}_{h}$ and $\Omega$.

Inserting (3.13) and (3.14) into (3.12) yields the assertion of the theorem.

Copyright (c) by SIAM. Unauthorized reproduction of this article is prohibited. 

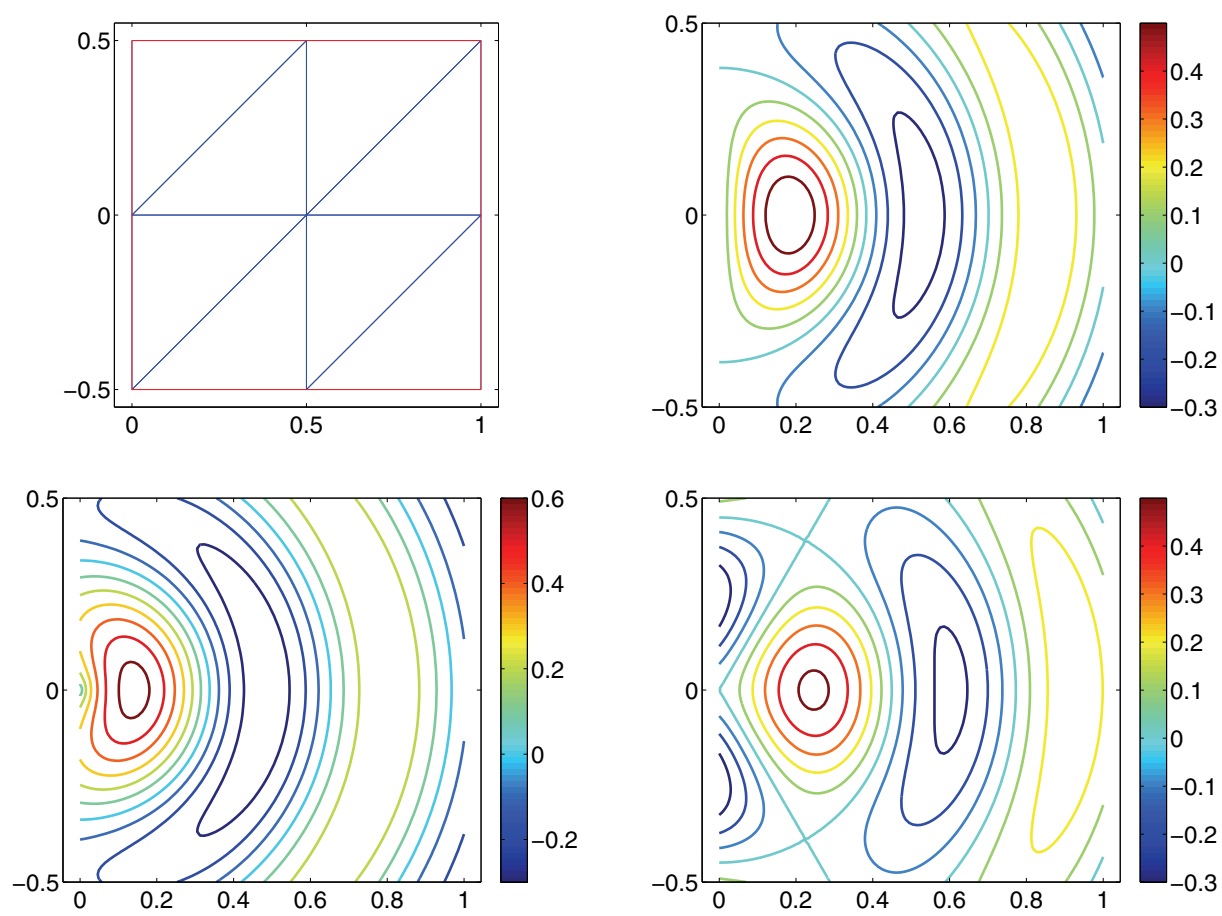

FIG. 4.1. The mesh used for the numerical experiments and the analytical solutions for $\xi=1$ (top right), $\xi=2 / 3$ (bottom left), $\xi=3 / 2$ (bottom right), and $\omega=10$.

4. Numerical experiments. In this section, we numerically investigate the $p$ convergence of the PWDG method for regular and singular solutions of the Helmholtz equation in two dimensions.

We consider a square domain $\Omega=[0,1] \times[-0.5,0.5]$, partitioned by a mesh consisting of eight triangles (see Figure 4.1, upper-left plot), so that $h=1 / \sqrt{2}$. For the time being, we fix $\omega=10$, such that an entire wavelength $\lambda=2 \pi / \omega \simeq 0.628$ is completely contained in $\Omega$. All of the computations have been done in MATLAB, and the system matrix was computed by exact integration on the mesh skeleton.

We choose the inhomogeneous boundary conditions in such a way that the analytical solutions are the circular waves given, in polar coordinates $\boldsymbol{x}=(r \cos \theta, r \sin \theta)$, by

$$
u(\boldsymbol{x})=J_{\xi}(\omega r) \cos (\xi \theta), \quad \xi \geq 0 ;
$$

here, $J_{\xi}$ denotes the Bessel function of the first kind and order $\xi$. For $t \ll 1$, these functions behave like

$$
J_{\xi}(t) \approx \frac{1}{\Gamma(\xi+1)}\left(\frac{t}{2}\right)^{\xi}
$$

Thus, if $\xi \in \mathbb{N}, u$ can be analytically extended to a Helmholtz solution in $\mathbb{R}^{2}$, while, if $\xi \notin \mathbb{N}$, its derivatives have a singularity at the origin. Then $u \in H^{\xi+1-\epsilon}(\Omega)$ for every $\epsilon>0$, but $u \notin H^{\xi+1}(\Omega)$ (see [16, Theorem 1.4.5.3]). 

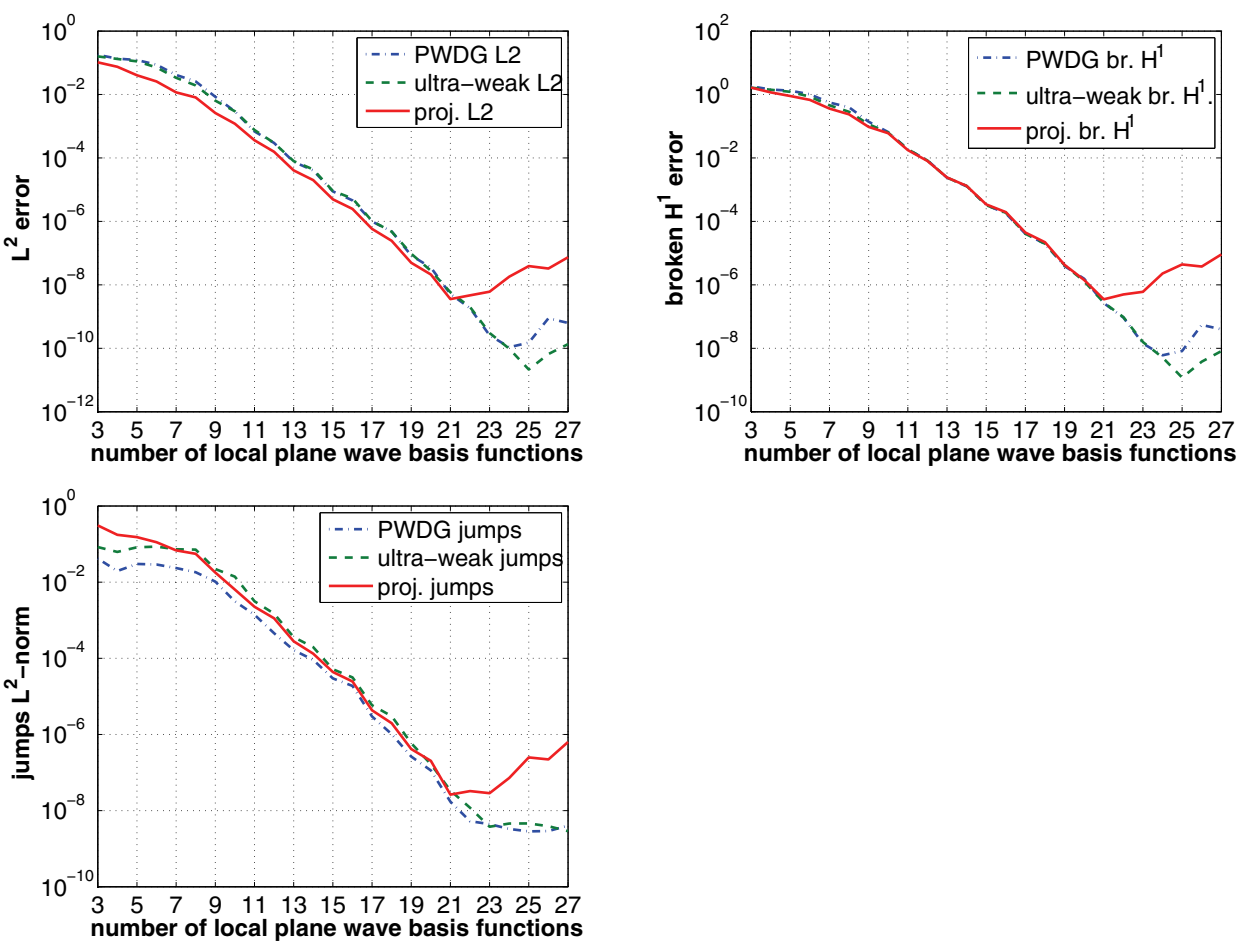

FIG. 4.2. The errors in $L^{2}$-norm, $H^{1}$-seminorm, and $L^{2}$-norm for the jumps for the regular solution $u=J_{1}(\omega r) \cos (\theta)$ plotted against $p \in\{3, \ldots, 27\}$. The convergence is exponential before the onset of numerical instability, and the discretization error is very close to the $L^{2}$-projection error.

We compute the numerical solutions in the regular case $\xi=1$ and in the singular cases $\xi=2 / 3$ and $\xi=3 / 2$. The profiles of the analytical solutions corresponding to these three cases are displayed in Figure 4.1, upper-right and lower plots.

We consider two choices of numerical fluxes: with constant parameters, as in the original ultra weak variational formulation (UWVF) of Cessenat and Després [11] $(\alpha=\beta=\delta=1 / 2$; dashed line in the plots), or depending on $p, h$, and $\omega$ as in Remark 3.16: $\alpha=\beta^{-1}=\delta^{-1}=\mathrm{a}_{0} p /(\omega h \log p)$, with $\mathrm{a}_{0}=10$ (PWDG from here on; dashed-dotted lines in the plots). We also plot the error of the $L^{2}$-projection of $u$ onto $V_{p}\left(\mathcal{T}_{h}\right)$ (solid line). For every case, we compute the $L^{2}$-norm of the error, the broken $H^{1}$-seminorm, and the $L^{2}$-norm of the jumps on the skeleton of the mesh. The errors are plotted in Figures 4.2-4.5.

These plots highlight three different regimes for increasing $p$ : (i) a preasymptotic region with slow convergence, (ii) a region of faster convergence, and finally, (iii) a sudden stalling of convergence, due to the impact of round-off. In fact, for high dimensional local bases, it has been observed that PWDG approaches suffer from serious ill-conditioning (see [11] and [25]); thus without an appropriate preconditioning or a clever choice of the bases it is impossible to obtain meaningful results for large $p$. Since implementation difficulties are not the focus of this paper, we refer only to [25] for a discussion of this issue and a possible remedy.

With a parameter $\mathrm{a}_{0} \geq 5$ in the definition of the fluxes, such that the condition $\delta<1$ (and thus $1-\delta>0$ ) is satisfied for all the considered $p$, the PWDG method is slightly superior to the one with constant fluxes (UWVF) in the $L^{2}$ - and $H^{1}$-norms; the difference in the jump norm is even more pronounced. 

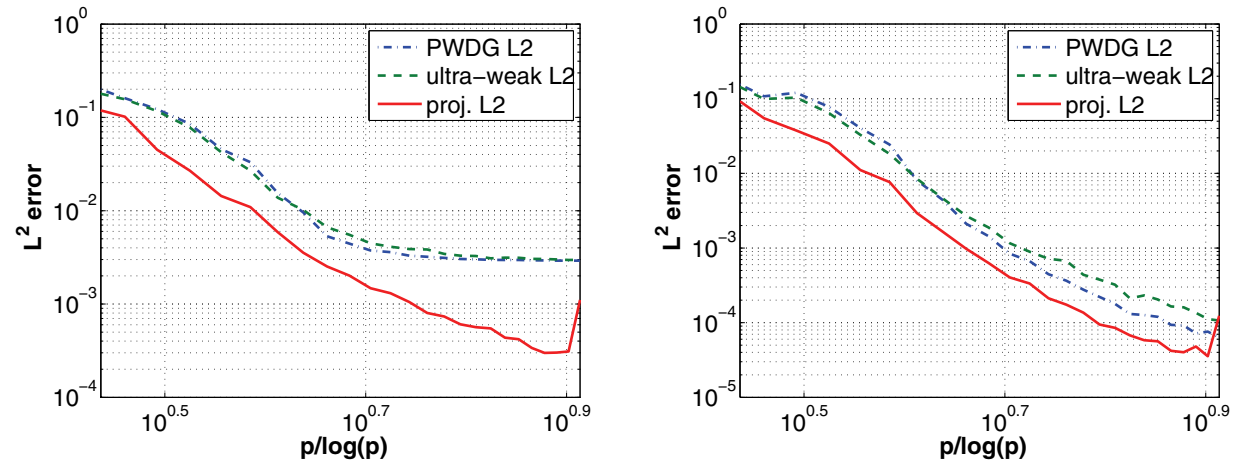

FIG. 4.3. The errors in $L^{2}$-norm for the two singular solutions $(\xi=2 / 3$ on the left and $\xi=3 / 2$ on the right) in logarithmic scale with respect to $p / \log p, p \in\{3, \ldots, 27\}$.
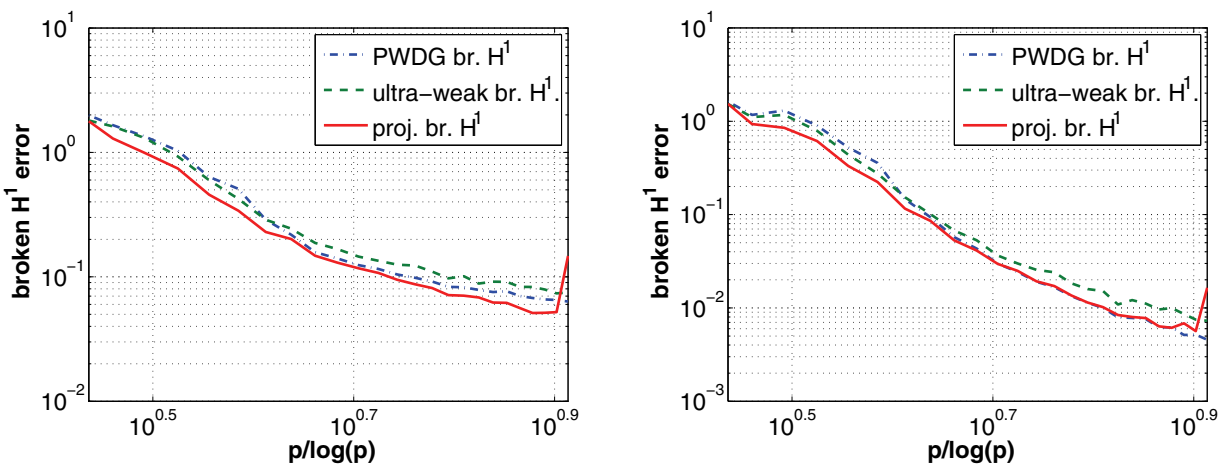

FIG. 4.4. The errors in broken $H^{1}$-seminorm for the two singular solutions ( $\xi=2 / 3$ on the left and $\xi=3 / 2$ on the right) in logarithmic scale with respect to $p / \log p, p \in\{3, \ldots, 27\}$.
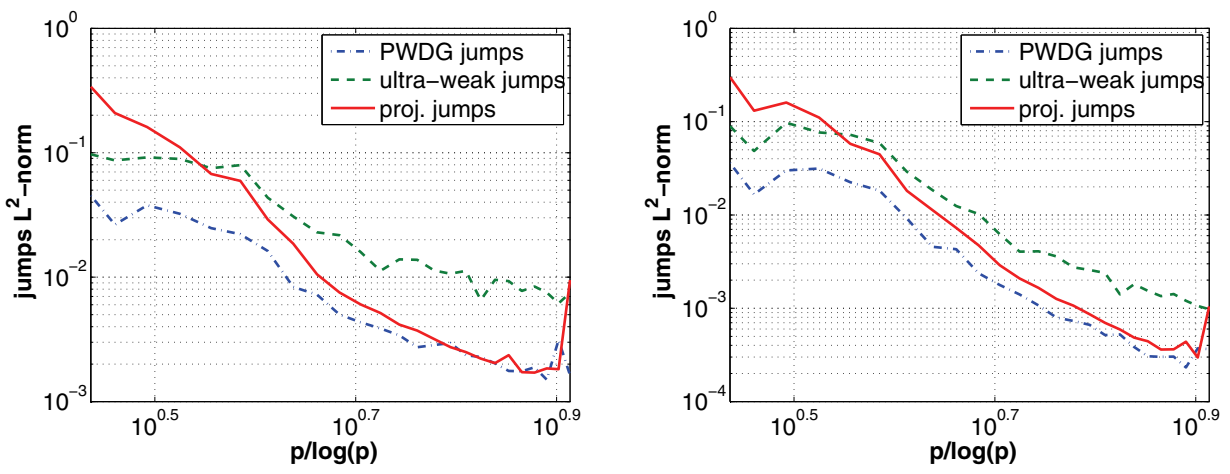

Fig. 4.5. The errors in $L^{2}$-norm on the skeleton for the jumps of the two singular solutions ( $\xi=2 / 3$ on the left and $\xi=3 / 2$ on the right) in logarithmic scale with respect to $p / \log p, p \in$ $\{3, \ldots, 27\}$.

The most evident outcome is that, for both methods, the numerical errors are always close to $L^{2}$-approximation error of the analytical solution; that is, the $p$-version is not affected by the pollution effect.

Copyright (c) by SIAM. Unauthorized reproduction of this article is prohibited. 

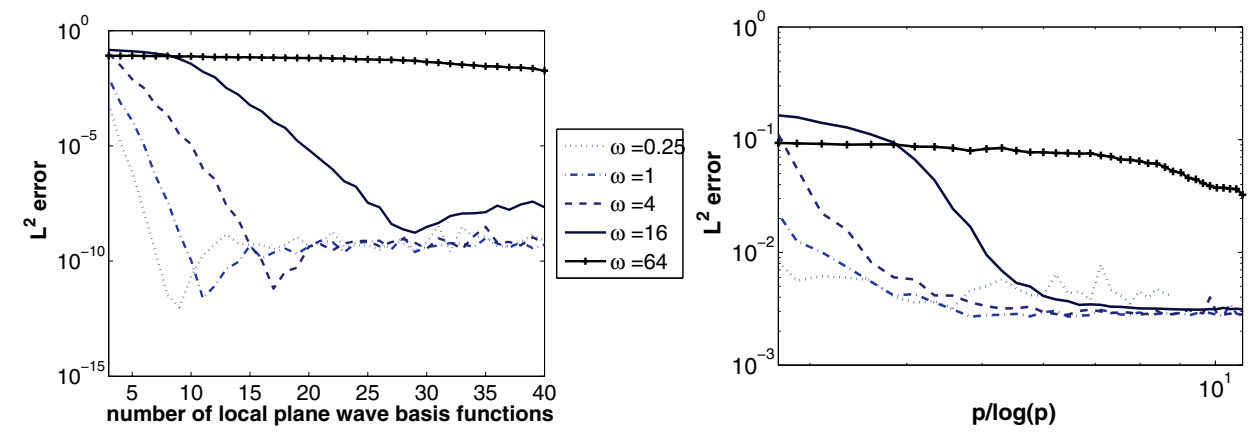

FIG. 4.6. The errors in $L^{2}$-norm for the regular solution $(\xi=1$, on the left) and the singular one $(\xi=2 / 3$, on the right, in logarithmic scale with respect to $p / \log p)$ for different values of $\omega(0.25,1,4,16,64), p \in\{3, \ldots, 40\}$.

The discretization error for $\xi=1$ (analytic solution) converges in all the considered norms with exponential rate (see Figure 4.2). This behavior is not a surprise: the algebraic convergence in the theoretical estimates is due only to the best approximation error and becomes exponential when the analytical solution of the problem can be extended analytically outside the domain (see Remark 3.14).

For $\xi=2 / 3$ and $\xi=3 / 2$, the solution $u$ has a singularity located in a boundary node of the mesh. It corresponds to the typical corner singularities arising from reentrant corners in scattering problems. In this case, as expected, the convergence is not exponential but algebraic, although the orders of convergence are not clear. In the region of faster convergence, the orders are significantly better than the ones expected from the theory; for higher $p$, numerical instability prevents us from obtaining a neat slope in the logarithmic plot. In all the considered norms, the orders of convergence are clearly better for the solution with higher Sobolev regularity (with $\xi=3 / 2$, $u \in H^{2}(\Omega)$ ).

By decreasing the wavenumber $\omega$, keeping the mesh fixed, we obtain a faster convergence in all the norms for both methods; see Figure 4.6. On the other hand, the instability appears for smaller $p$ because the plane waves are closer to being linearly dependent. Of course in this case the domain accommodates fewer wavelengths.

Conversely, if we increase $\omega$, again with the same mesh, the preasymptotic region becomes larger and larger (more plane waves are needed before the onset of convergence) and the instability reduces the maximum possible accuracy we can reach.

5. Conclusions. We have presented the first $p$-version a priori error analysis of the plane wave discontinuous Galerkin (PWDG) method for the 2D homogeneous Helmholtz equation. Quasi-optimal error estimates in an energy-type norm are established by using a modified duality argument due to [10,29]. On the other hand, our analysis does not provide an estimate of the gradient of the error (we were able to only estimate the gradient of the difference between the analytical solution and a computable projection of the PWDG solution), or a quasi-optimal estimate of the $L^{2}$-norm of the error. These problems remain open.

As far as the 3D case is concerned, the projection error estimates for solutions to the homogeneous Helmholtz equation onto plane wave spaces proved in [19] and the regularity results of $[12,17]$ allow us to generalize the analysis carried out in this paper to the 3D setting. 
Acknowledgment. The authors wish to thank Claude Gittelson for providing his MATLAB implementation of PWDG as a basis for the numerical experiments.

\section{REFERENCES}

[1] M. Ainsworth, Discrete dispersion relation for hp-version finite element approximation at high wave number, SIAM J. Numer. Anal., 42 (2004), pp. 563-575.

[2] M. Ainsworth, Dispersive and dissipative behaviour of high order discontinuous Galerkin finite element methods, J. Comput. Phys., 198 (2004), pp. 106-130.

[3] M. Ainsworth, Dispersive properties of high-order Nédélec/edge element approximation of the time-harmonic Maxwell equations, Philos. Trans. R. Soc. Lond. Ser. A Math. Phys. Eng. Sci., 362 (2004), pp. 471-491.

[4] M. Amara, R. Djellouli, and C. Farhat, Convergence analysis of a discontinuous Galerkin method with plane waves and Lagrange multipliers for the solution of Helmholtz problems, SIAM J. Numer. Anal., 47 (2009), pp. 1038-1066.

[5] I. BabušKa And J. M. Melenk, The partition of unity method, Internat. J. Numer. Methods Engrg., 40 (1997), pp. 727-758.

[6] I. M. BABUŠKa AND S. A. SAUter, Is the pollution effect of the FEM avoidable for the Helmholtz equation?, SIAM Rev., 42 (2000), pp. 451-484.

[7] I. BABUŠKA AND M. SURI, The $p$ and $h p$ versions of the finite element method, basic principles and properties, SIAM Rev., 36 (1994), pp. 578-632.

[8] T. Betcke, Numerical Computation of Eigenfunctions of Planar Regions, Ph.D. thesis, University of Oxford, Oxford, UK, 2005.

[9] S. C. Brenner and L. R. Scott, The Mathematical Theory of Finite Element Methods, 2nd ed., Texts Appl. Math. 15, Springer-Verlag, New York, 2002.

[10] A. Buffa ANd P. Monk, Error estimates for the ultra weak variational formulation of the Helmholtz equation, M2AN Math. Model. Numer. Anal., 42 (2008), pp. 925-940.

[11] O. Cessenat And B. Després, Application of an ultra weak variational formulation of elliptic PDEs to the two-dimensional Helmholtz problem, SIAM J. Numer. Anal., 35 (1998), pp. 255-299.

[12] P. Cummings And X.-B. Feng, Sharp regularity coefficient estimates for complex-valued acoustic and elastic Helmholtz equations, Math. Models Methods Appl. Sci., 16 (2006), pp. 139160.

[13] C. Farhat, R. Tezaur, and P. Weidemann-Goiran, Higher-order extensions of a discontinuous Galerkin method for mid-frequency Helmholtz problems, Internat. J. Numer. Methods Engrg., 61 (2004), pp. 1938-1956.

[14] G. GABARD, Discontinuous Galerkin methods with plane waves for time-harmonic problems, J. Comput. Phys., 225 (2007), pp. 1961-1984.

[15] C. J. Gittelson, R. Hiptmair, and I. Perugia, Plane wave discontinuous Galerkin methods: Analysis of the h-version, M2AN Math. Model. Numer. Anal., 43 (2009), pp. 297-331.

[16] P. Grisvard, Elliptic Problems in Nonsmooth Domains, Monogr. Stud. Math. 24, Pitman, Boston, 1985.

[17] U. Hetmaniuk, Stability estimates for a class of Helmholtz problems, Commun. Math. Sci., 5 (2007), pp. 665-678.

[18] R. Hiptmair, A. Moiola, and I. Perugia, Approximation by Plane Waves, Technical report 2009-27, SAM-ETH Zürich, Zürich, Switzerland, 2009.

[19] R. Hiptmair, A. Moiola, and I. Perugia, Approximation by Plane Waves, ZAMP, submitted.

[20] R. Hiptmair and I. Perugia, Mixed plane wave discontinuous Galerkin methods, in Domain Decomposition Methods in Science and Engineering XVIII, M. Bercovier, M. J. Gander, R. Kornhuber, and O. Widlund, eds., Lect. Notes Comput. Sci. Eng., Springer, Berlin, Heidelberg, 2008, pp. 51-62.

[21] T. Huttunen, P. Gammallo, and R. J. Astley, Comparison of two wave element methods for the Helmholtz problem, Comm. Numer. Methods Engrg., 25 (2009), pp. 35-52.

[22] T. Huttunen, J. P. Kaipio, And M. Malinen, Optimal control in high intensity focused ultrasound surgery, in Optimization in Medicine, Springer Optim. Appl. 12, C. J. S. Alves, P. M. Pardalos, and L. N. Vicente, eds., Springer, New York, 2008, pp. 169-195.

[23] T. Huttunen, M. Malinen, And P. Monk, Solving Maxwell's equations using the ultra weak variational formulation, J. Comput. Phys., 223 (2007), pp. 731-758.

[24] T. Huttunen And P. Monk, The use of plane waves to approximate wave propagation in anisotropic media, J. Comput. Math., 25 (2007), pp. 350-367.

Copyright $@$ by SIAM. Unauthorized reproduction of this article is prohibited. 
[25] T. Huttunen, P. Monk, And J. P. Kaipio, Computational aspects of the ultra-weak variational formulation, J. Comput. Phys., 182 (2002), pp. 27-46.

[26] J. Melenk, On Generalized Finite Element Methods, Ph.D. thesis, University of Maryland, College Park, MD, 1995.

[27] J. M. Melenk, Operator adapted spectral element methods I. Harmonic and generalized harmonic polynomials, Numer. Math., 84 (1999), pp. 35-69.

[28] A. Moiola, Approximation Properties of Plane Wave Spaces and Application to the Analysis of the Plane Wave Discontinuous Galerkin Method, Technical report 2009-06, SAM-ETH Zürich, Zürich, Switzerland, 2009.

[29] P. Monk And D. Q. Wang, A least squares method for the Helmholtz equation, Comput. Methods Appl. Mech. Engrg., 175 (1999), pp. 121-136.

[30] E. Perrey-Debain, O. Laghrouche, and P. Bettess, Plane-wave basis finite elements and boundary elements for three-dimensional wave scattering, Philos. Trans. R. Soc. Lond. Ser. A Math. Phys. Eng. Sci., 362 (2004), pp. 561-577.

[31] H. Riou, P. LadevÉze, and B. Sourcis, The multiscale VTCR approach applied to acoustics problems, J. Comput. Acoust., 16 (2008), pp. 487-505.

[32] R. Tezaur and C. FARhat, Three-dimensional discontinuous Galerkin elements with plane waves and Lagrange multipliers for the solution of mid-frequency Helmholtz problems, Internat. J. Numer. Methods Engrg., 66 (2006), pp. 796-815.

Copyright (c) by SIAM. Unauthorized reproduction of this article is prohibited. 